\title{
WILEY-VCH
}

\section{Liquid Crystalline Vitrimers with Full or Partial Boronic-Ester Bond Exchange}

Mohand O. Saed, Alexandra Gablier and Eugene M. Terentjev*

Dr. M. O. Saed, A. Gablier and Prof. E. M. Terentjev

Cavendish Laboratory, University of Cambridge, J.J. Thomson Avenue, Cambridge, CB3

OHE, United Kingdom

E-mail: emt1000@cam.ac.uk

Keywords: liquid crystalline elastomers, vitrimers, boronic-ester, actuators, bilayer

In this manuscript, a new vitrimer chemistry strategy (boronic transesterification) is introduced into liquid crystal elastomers (LCE) to allow catalyst-free bond exchange, to enable processing (director alignment, remolding, and welding) in the liquid crystalline (nematic) phase. Additionally, the concept of partial vitrimer network is explored, where a percolating fraction of the network remains permanently crosslinked, hence preserving the integrity of the materials and preventing large creep. This combined strategy allows us to avoid the shortcomings of current methods of aligning LCE, especially in complex shapes. Thiol-acrylate Michael addition reaction is used to produce uniform polymer networks with controllable thermomechanical response and local plasticity. Control of the plasticity is achieved by varying the fractions of permanent and exchangeable network, where a material 'sweet spot' with an optimum elastic/plastic balance is identified. Such exchangeable LCE (xLCE) allows postpolymerization processing, while also minimizing unwanted creep during actuation. Moreover, conjoining multiple materials (isotropic and liquid-crystalline) in a single covalently-bonded composite structure results in a variety of smart morphing systems that adopt shapes with complex Gaussian curvature. Remolding and welding xLCEs may enable the applications of these materials as mechanical actuators in reversibly folding origamis, in-vivo artificial muscles, and in soft robotics. 


\section{WILEY-VCH}

\section{Introduction}

The idea of a large-strain reversible mechanical actuator based on the intrinsic material properties of liquid-crystalline elastomers (LCE) has been understood for over 20 years. $^{[1-3]}$ Key characteristics of LCE actuation are remarkable: fully reversible action, ${ }^{[4]}$ large-amplitude, with a stroke of up to $500 \%,{ }^{[5,6]}$ and the stress-strain-speed response almost exactly matching the human muscle. ${ }^{[7]}$ These properties make LCE highly attractive in biomedical engineering, robotics, smart textiles, and many other fields. Yet, there is a profound difficulty: a bottleneck, which remained the reason why this concept has not yet found its way into many practical devices and applications. LCE actuation requires alignment of the local orientational anisotropy in the permanently crosslinked polymer network (monodomain structure), which has been impossible to achieve in any useful large-scale configuration other than in a flat film, due to the unavoidable restrictions of two competing processes: orientational alignment and network crosslinking. The classical two-step procedure of Finkelmann ${ }^{1,2}$ has long remained the only method, utilized even in the more modern fiber-drawing or 3Dprinting setting. ${ }^{[5,8]}$ Recently, a breakthrough has been achieved by combining the LCE structure with the 'vitrimer' concept of a polymer network in which some of the covalent bonds are capable of bond-exchange reaction (BER), leading to the 'exchangeable LCE' concept (xLCE). ${ }^{[9]}$ Vitrimers are much more stable than transient (thermoplastic) networks held by physical crosslinking, but also allow easy thermal re-molding (making the material fully renewable), including molding of complex shapes with intricate local alignment (which is difficult in traditional LCE relying on permanent crosslinking). However, it turns out that the continuous availability of the thermally-induced BER in the xLCE network makes the temperature range of actuation limited: as soon as the temperature is elevated towards the vitrification point, the material creep sets in. The 


\section{WILEY-VCH}

same problem has been shown to affect the xLCE alignment stage, achieved in a vitrimer by setting a uniaxial creep flow under stress. ${ }^{[9,10]}$ Recently, Wen et al. developed an interesting class of xLCE with exchangeable bonds based on polyurethane (bonds of carbamates to isocyanates and alcohols at elevated temperature). ${ }^{[1]}$ Nevertheless, the creep problem associated with thermally induced bond exchange has not been addressed by this chemistry strategy either. Light-induced dynamic bonds, such as disulfide and free-radical addition-fragmentation chain transfer (AFT), have been also used to align the LCE networks by application of UV light at room temperature. ${ }^{[12,13]}$ However, disulfide-based LCE also suffers from creep at elevated temperature due to the thermally induced residual disulfide bond exchange. ${ }^{[14]}$ Although AFT is shown to be an effective mechanism to align LCE, it only works for a few programming cycles due to the photoinitiator degradation or the termination reaction. In this paper we show that creating an xLCE network, with partially permanent and partially exchangeable strands, could address both these difficulties (achieving alignment and eliminating the residual plastic flow) on the way to developing robust actuators with complex shapes.

Network plasticity can be obtained in two ways: either through BER, or through bond cleavage and re-forming. ${ }^{[15]}$ These latter reactions are based on the concept of chemical equilibrium in covalent adaptable networks (CAN) - or physical equilibrium in thermoplastic networks, which works on the same principle. Examples of CAN reactions are: Diels-Alder, ${ }^{[16]}$ disulfide metathesis. ${ }^{[12,17,18]}$, ART, ${ }^{[19]}$ thiol-thioester exchange. ${ }^{[20]}$ Vitrimers are a subset of CAN, defined as "a polymer network that can change its topology while maintaining the constant number of chemical bonds". ${ }^{[21-23]}$ A number of reaction strategies, such as transesterification, ${ }^{[21,24]}$ transamidation, ${ }^{[25]}$ transalkylation of triazolium salts, ${ }^{[26]}$ transcarbamoylation ${ }^{[27,28]}$ imine-amine exchange, ${ }^{[29]}$ siloxane exchange, ${ }^{[30]}$ and olefin metathesis ${ }^{[31]}$, have been employed to form covalent bondexchangeable networks. In these systems, a catalyst or an elevated temperature is needed 


\section{WILEY-VCH}

to enable the polymeric network to shuffle its topology. Recently, the concept of boronic cross-coupling (well-studied in small molecule synthesis ${ }^{[32-34]}$ ) has been applied to polymers. ${ }^{[35,36]}$ Unlike other dynamic bond systems, boronic-based exchanges do not need a catalyst. The reversible boronic-based bond exchange can occur by boronic transesterification ${ }^{[37,38]}$ or boroxine reversible bonds. ${ }^{[39,40]}$ Boronic transesterification is much more interesting for preparing vitrimers due to the strength and kinetic tunability of the switchable $\mathrm{B}-\mathrm{O}$ bonds. The readiness of the $\mathrm{B}-\mathrm{O}$ for exchange depends on the nature of the polymer network that includes these bonds (that is, semi-crystalline, glassy, or elastomeric). The bond exchange requires chain mobility and low energy barriers, so it could only occur above the melt $\left(\mathrm{T}_{\mathrm{m}}\right)$ or the glass $\left(\mathrm{T}_{\mathrm{g}}\right)$ transitions. ${ }^{[22,38,41]}$ Recent work from Cash et al. ${ }^{[41]}$ has demonstrated that the mechanical behavior of elastomers with boronic ester moieties can be controlled through the selective placement of static (irreversible) bonds between crosslinking points to complement the exchangeable bonds. The increase in the static bond is shown to improve the mechanical strength and reduce the ability of the materials to creep and relax the stress.

It is often required to process LCE (e.g. align, remold, weld) at low temperatures (in the nematic phase): to enable better alignment of the nematic director, or preserve this alignment while remolding or welding. Aligning LCE at a high temperature (in the isotropic phase) is a delicate process, which produces the "isotropic-genesis" LCE, but may also lead to fracture of samples due to the internal stress generated by the subsequent phase change. Up-to-date, all the thermally-induced bond-exchangeable xLCE have been processed in the isotropic phase despite the issues with the mesogen stability. ${ }^{[42]}$

Here, we introduce a transesterification based on boronic esters, to impart catalyst-free bond exchange to enable the molecular alignment and remolding/welding during the BER at a low temperature, in the nematic phase. We also introduce the concept of a partial vitrimer network, 


\section{WILEY-VCH}

where a percolating fraction of the network remains permanently crosslinked, thus preserving the integrity of the material and minimizing creep.

\section{Results and discussion}

The purpose of this study is to utilize the concept of partial vitrimer network to design xLCEs where the vitrimer behavior of the network can be used to process the materials (i.e. alignment, remolding and welding). We are guided by the recent work on using the thiol-acrylate reaction to design LCE with uniform and highly controllable network structures. $^{[43-45]}$ We sought to examine a one-pot thiol-acrylate "click" reaction to formulate malleable materials with a controllable degree of plastic flow and tunable processability post crosslinking. In our materials the plastic flow is controlled by changing the fraction of the boronic ester groups. A dithiol functionalized boronic ester monomer 2,2'-(1, 4-Phenylene)-bis[4-mercaptan-1,3,2-dioxaborolane] (BDB) is used as a chain extender, alongside the flexible dithiol monomer 2,2'(Ethylenedioxy)diethanethiol) EDDT (Scheme 1a). BDB is synthesized according to a

a)
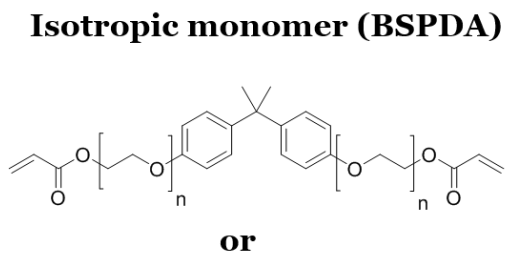

LC monomer (RM82)

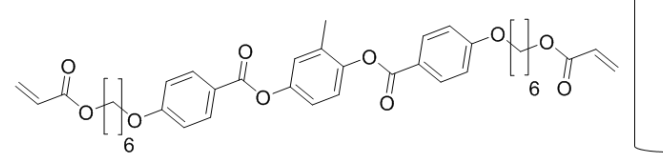

Bond-exchange spacer (BDB)

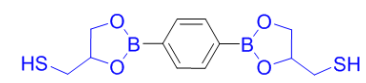

Flexible spacer (EDDT)

$$
\mathrm{SH}_{\mathrm{O}} \widehat{\mathrm{O}} \widehat{\mathrm{SHH}}_{\mathrm{SH}}
$$

Crosslinker (PETMP)

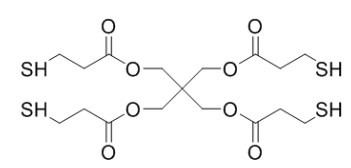

b)
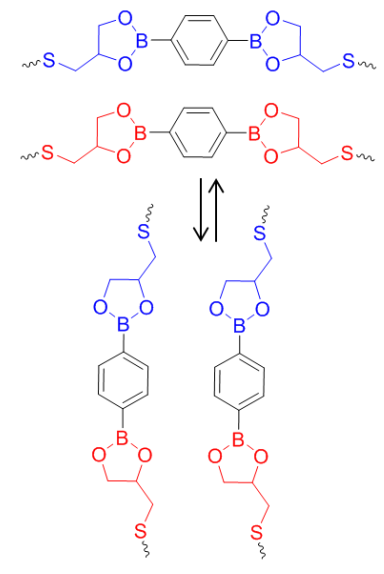

Scheme 1. Materials used in the study. a) Chemical structures of diacrylate, dithiols, and crosslinker monomers. Isotropic and LCE networks are formulated with various boronic ester concentrations through Michael addition. BSPDA is used to produce the isotropic networks where RM82 is used to create the mesogenic order. b) Boronic ester exchange reaction through transesterification mechanism. 


\section{WILEY-VCH}

method previously described by Chan et al. (Figure S1, Supporting Information). ${ }^{[46]}$ Polymer networks were produced following a modified procedure described by Saed at al. ${ }^{[47]}$ While keeping the 1:1 stoichiometric ratio between thiol and acrylate groups, and the amount of the permanent 4-functional crosslinking constant $(0.3$ molar ratio of PETMP, counted by reactive bonds), while the choice of the thiol spacers ( 0.7 molar ratio, by reacting bonds) is varied between $\mathrm{BDB}$ and EDDT to carefully control the degree of the possible plastic flow. The boronic transesterification mechanism of the plastic flow is illustrated in Scheme $\mathbf{1 b .}$

Table 1 Chemical formulations and monomers mass ratio for isotropic and LCE systems used. All systems are synthesized using thiol-acrylate Michael addition reaction using an equal amount of catalyst, $1 \mathrm{wt} \%$ Trimethylamine (TEA) and $40 \mathrm{wt} \%$ Tetrahydrofuran (THF) solvent.

\begin{tabular}{llllll}
\hline $\begin{array}{l}\text { Network } \\
\text { description }\end{array}$ & $\begin{array}{l}\text { Mass of } \\
\text { BSPDA }\end{array}$ & $\begin{array}{l}\text { Mass } \\
\text { of RM82 }\end{array}$ & $\begin{array}{l}\text { Mass of } \\
\text { PETMP }\end{array}$ & $\begin{array}{l}\text { Mass of } \\
\text { EDDT }\end{array}$ & $\begin{array}{l}\text { Mass of } \\
\text { BDB }\end{array}$ \\
\hline 0 BDB & & & & & \\
ISO & 1 & 0 & 0.157 & 0.273 & 0 \\
LCE & 0 & 1 & 0.112 & 0.195 & 0 \\
\hline 5 BDB ISO & 1 & 0 & 0.157 & 0.259 & 0.025 \\
\hline 10 BDB ISO & 1 & 0 & 0.157 & 0.245 & 0.051 \\
\hline 15 BDB & & & & & \\
ISO & 1 & 0 & 0.157 & 0.232 & 0.076 \\
LCE & 0 & 1 & 0.112 & 0.166 & 0.054 \\
\hline 25 BDB ISO & 1 & 0 & 0.157 & 0.205 & 0.126 \\
\hline 100 BDB & & & & & \\
ISO & 1 & 0 & 0.157 & 0 & 0.506 \\
LCE & 0 & 1 & 0.112 & 0 & 0.362 \\
\hline
\end{tabular}

To compare and contrast the effects of bond-exchange plastic deformation and the separate slow relaxation of the nematic order, we prepared the matching isotropic and mesogenic networks. The isotropic networks are formulated using 1 molar ratio of isotropic acrylate monomer, Biphenyl A ethoxylate diacrylate (BSPDA), reacting with multifunctional thiols: 0.3 molar ratio 4-thiol crosslinker, Pentaerythritol tetrakis(3- 


\section{WILEY-VCH}
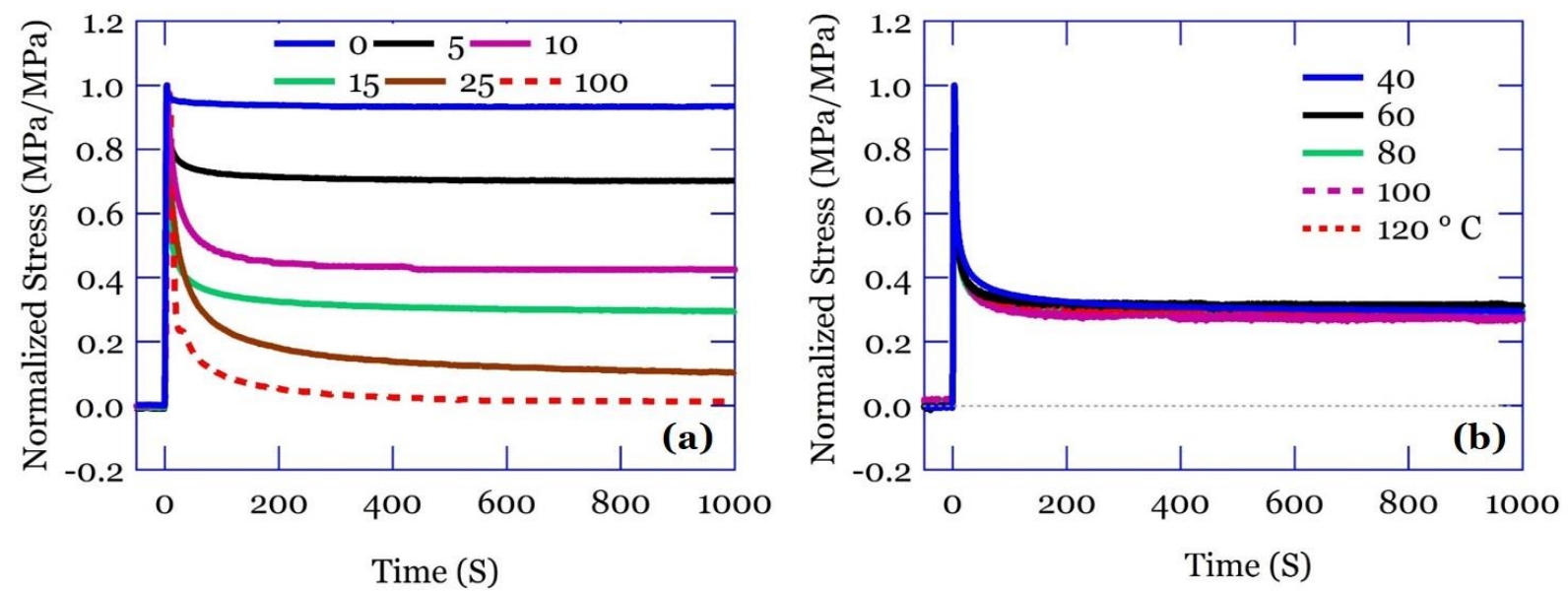

Figure 1. Influence of boronic ester concentration on stress relaxation behavior of isotropic networks. a) Effect of boronic ester concentration on relaxation rate and equilibrium stress for $0,5,10,15,25$, and 100BDB networks, samples were tested under constant uniaxial strain $3 \%$ imposed at $\mathrm{t}=0$, at $40{ }^{\circ} \mathrm{C}$. b) Stress relaxation does not depend on temperature, the example shown is for 15BDB network.

mercaptopropionate) (PETMP) and 0.7 molar ratio of chain extenders BDB and EDDT. Varying the ratio of BDB to EDDT results in different fractions of the exchangeable network part. The mass ratio, and the description of each formulation are given in Table 1. Stress relaxation is a widely used method to characterize the degree of plasticity in polymer networks. We were informed by a previous study showing that a permanent crosslinked LCE can relax the stress very significantly in the nematic phase due to the rearrangements of mesogens. ${ }^{[48]}$ To isolate the stress relaxation caused by boronic ester bond exchange from the relaxation due to director rearrangement in LCE network, we initially studied the stress relaxation behavior of boronic ester-containing polymers using the isotropic network model, Figure 1. It has been demonstrated that the stress relaxation in a bond-exchange network is characterized by a single rate of the reaction, resulting in the simple-exponential law of relaxation ${ }^{[24,49]}$ (this is in stark contrast with thermoplastic elastomers where a broad distribution of relaxation rates leads to a highlystretched exponential relaxation of stress $\left.{ }^{[49]}\right)$. Hence in a vitrimer network the relaxation time obtained by the exponential fitting of data is the inverse of the reaction rate. The 


\section{WILEY-VCH}

stress relaxation is tested after instantaneously imposing a constant strain of $3 \%$, at temperatures well above their $\mathrm{T}_{\mathrm{g}}$. Differential scanning calorimetry (DSC) plots showing thermal transitions of these materials can be found in Figure. S2 in the Supporting Information. As shown in Figure 1a, the sample with the highest BDB concentration (100BDB) relaxes its stress to zero within a few seconds. Upon decreasing the BDB content, the samples resist the complete stress relaxation, due to the increasing fraction of the permanent network. For a network without any boronic ester (OBDB), the stress remains almost a constant after a few fractions of a second. Note that in samples with moderate amount of $\mathrm{BDB}$, for instance $15 \mathrm{BDB}$, the stress relaxation rate is similar to that of the fully plastic 100BDB sample, while the equilibrium stress never reaches zero. In other words, the stress relaxation of $15 \mathrm{BDB}$ is the additive combination of the plastic response of a true vitrimer (like 100BDB) and the equilibrium elastic response of a true thermoset $(\mathrm{OBDB})$. The residual equilibrium stress is highly tunable via adjusting the amount of boronic ester (Figure. S3). As an example of a network with partial dynamic bonds, 15BDB was selected to further investigate the temperature dependence of the relaxation behavior. Unexpectedly, the stress relaxation behavior has very little temperature dependence when samples are tested above $\mathrm{T}_{\mathrm{g}}$ (Figure 1b). The lack of temperature dependence in the rate of bond exchange is a difficult and a somewhat controversial point. This has to be attributed to the low energy barriers of boronic ester bonds; heating above $\mathrm{T}_{\mathrm{g}}$ is enough to overcome these barriers and facilitates rapid bond exchanges. It suggests that the observed relaxation time of a few seconds is determined by the internal network dynamics, not the bond exchange activation. This fact is actually confirmed in the early work of Sumerlin, ${ }^{[37,41]}$ while in the key paper by Leibler ${ }^{[22]}$ the authors claimed to see a temperature dependence of their relaxation time, and even determined the activation energy from that. However, a close examination of their data suggests that this was probably not the case: their relaxation curves at different 


\section{WILEY-VCH}
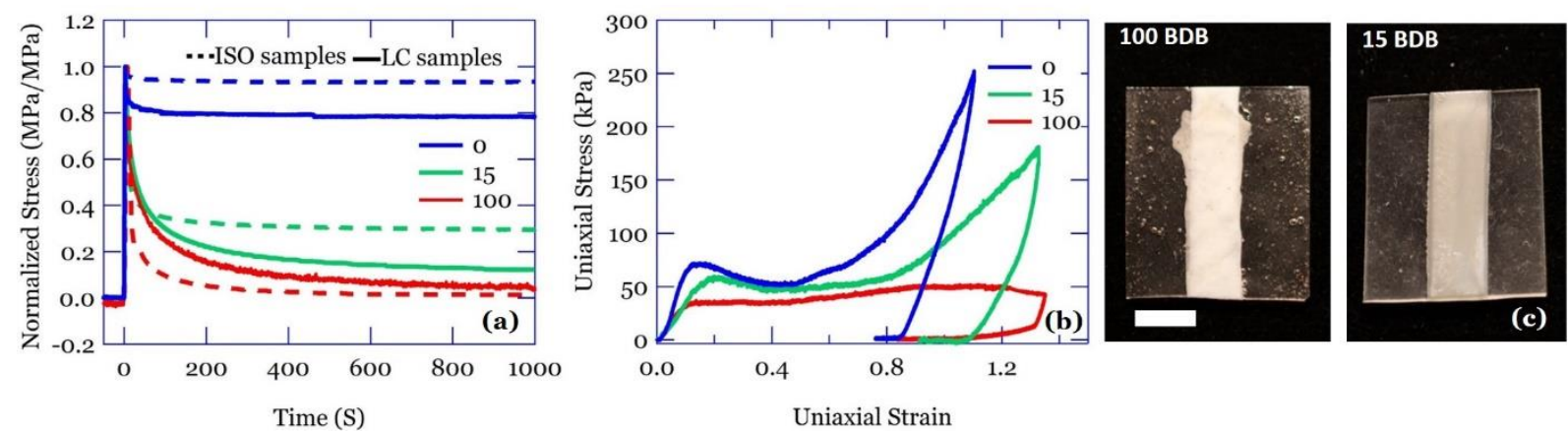

Figure 2. Stress relaxation, stress-strain, and welding of full (100BDB) and partial (15BDB) vitrimers, and a thermoset (OBDB). a) Comparison of stress relaxation of isotropic (dashed lines) and liquid crystal (solid lines) networks, all samples tested under similar conditions at $80^{\circ} \mathrm{C}$. b) Effect of boronic ester concentration on stress-strain behavior for liquid crystal materials, samples tested in the nematic phase at $20^{\circ} \mathrm{C}$ below $\mathrm{T}_{\text {ni. }}$ c) Images of isotropic (transparent) and liquid crystal (opaque) films showing the welding of fully (100BDB) and partly (15BDB) vitrimer materials (see text for detail). Scale bar: $5 \mathrm{~mm}$.

temperatures actually match each other, and the difference is probably due to the ambiguity in defining the $t=0$ point on a log-scale between the curves.

Next, we prepared xLCE networks with variable boronic ester concentration, and preformed stress relaxation experiments on these. Comparison of stress relaxation behavior of LCE (solid lines) with the corresponding isotropic materials (dashed lines) is shown in Figure 2a. All the studied samples (100, 15, and 0BDB) were tested under similar test conditions at $80^{\circ} \mathrm{C}$ to ensure all the isotropic materials are rubbery, and all LCE in the nematic phase. As expected, LCE have relaxed the stress slower than isotropic materials due to their dual relaxation mechanism: apart from the bond exchange, LCEs can also relax the stress via nematic director rearrangement. ${ }^{[48]}$ The relaxation through the latter mechanism can be clearly seen in the OBDB LCE network: despite there not being any exchangeable bonds, the LCE still strongly relaxes its stress, 


\section{WILEY-VCH}

whereas in the similar isotropic material the relaxation was insignificant past the first fractions of a second.

The plastic flow of the xLCE materials was further investigated by deforming the samples isothermally in the nematic phase $\left(20^{\circ}\right.$ below $\left.\mathrm{T}_{\mathrm{ni}}\right)$ at a low strain rate of $\sim 0.002 \mathrm{~s}^{-}$ ${ }^{1}$ (Figure 2b). The xLCE sample with the highest boronic ester concentration shows a typical stress-strain curve of thermoplastic materials, as expected due to the large amount of exchangeable bonds. On the other hand, LCE without bond exchange (OBDB) showed a classical stress-strain response of polydomain LCE, with three characteristic regions: the linear elastic deformation below a threshold, the soft elasticity plateau during the director re-alignment, and the elastic deformation of a monodomain. ${ }^{[50,51]}$ xLCE with a partially exchangeable network (15BDB) has an interesting 'intermediate' stress-strain curve, similar to the traditional LCE, but with a significantly longer soft plateau. This has to be attributed to the rearrangement of boronic ester bonds, which acts as an additional dissipative mechanism, allowing for significant deformation without energy cost.

The presence of exchangeable boronic ester bonds offers a robust method to remold, and covalently weld or adhere diverse groups of polymers at a relatively low temperature. A demonstration showing permanent welding (covalent bonding) of isotropic and xLCE materials with full and partial vitrimer characteristics is presented in Figure 2c. In both cases, one views the sample from above, seeing the isotropic network as fully transparent on a black background, and the nematic xLCE network white in its polydomain state. In the first example, a $5 \times 18 \mathrm{~mm}$ xLCE strip with full vitrimer capacity (100BDB) is pressed with $15 \times 18 \mathrm{~mm}$ isotropic vitrimer (also 100BDB) between two Teflon blocks under mild conditions (weight of $500 \mathrm{~g}$ at $80{ }^{\circ} \mathrm{C}$ ). After $12 \mathrm{hr}$ the two films formed a single film with homogenous thickness, with full interpenetration of one 


\section{WILEY-VCH}
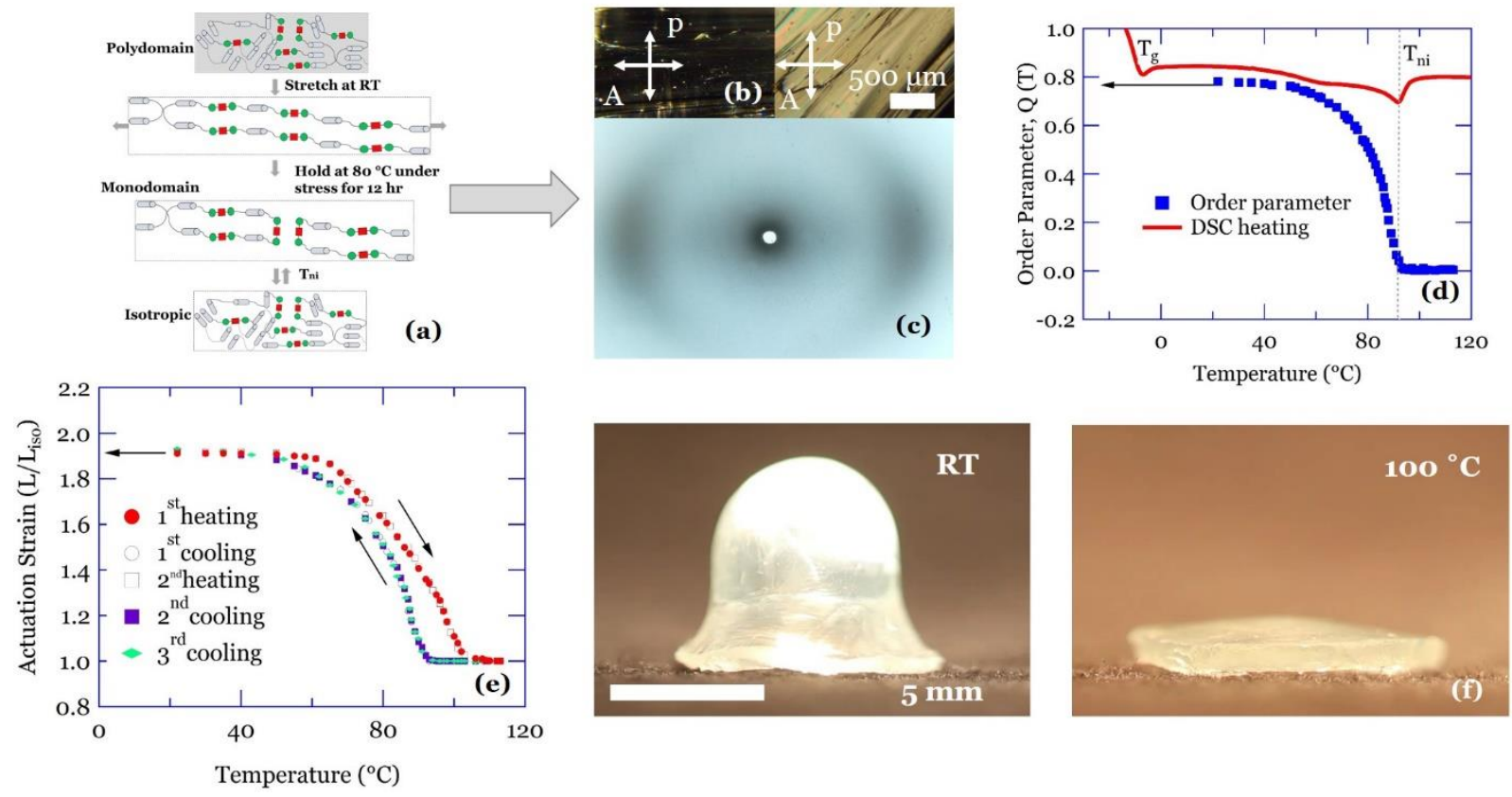

Figure 3. Programing 15BDB xLCE for actuation. a) Schematic showing the alignment process of the sample. b) Polarized optical micrographs viewing birefringence of a uniaxial aligned xLCE at $0^{\circ}$ (dark) and $45^{\circ}$ (bright) to the polarizer. c) Wide-angle x-ray scattering displaying 2D pattern for the uniaxial aligned sample. d) Order parameter (s) and heat flow as function of temperature for 15 BDB. e) Actuation strain for 2 heating and 3 cooling cycles of a uniaxially-aligned sample under a constant tensile load of $\sim 4 \mathrm{~g}(6 \mathrm{kPa})$. f) Side-view images of a dome-shaped $15 \mathrm{BDB}$ sample at room temperature (RT) and reversibly actuated at $100^{\circ} \mathrm{C}$.

materials into the other, and permanent bonding (fusing) between the two materials. In contrast, in the second image showing the partial vitrimer materials (15BDB), the two films also bonded into a single film, but with a distinct step in thickness, indicative of covalent bonding between the two materials but a lack of unrestricted plastic flow. It is clear that $\mathrm{OBDB}$ and 100BDB networks are not useful to create functional actuators, due to the lack of post polymerization processability in $\mathrm{OBDB}$, and the extreme plastic creep in $100 \mathrm{BDB}$.

A controlled fraction of exchangeable boronic ester bonds in an xLCE network also offers the ability to permanently align its director during the bond exchange in the nematic phase. We choose the 15BDB as the 'sweet spot' of the optimal balance between the high degree of remaining plasticity, and a still significant residual elastic modulus. 


\section{WILEY-VCH}

Our method of permanent alignment relies on the bond-exchange reaction, and the fair amount of the permanent bonds in the network. The original work of Pei et al. ${ }^{[9,10]}$ has relied on a delicate timing of initiating and stopping the uniaxial plastic flow, however, with the fully exchangeable network there will always be some creep on thermal actuation. In our case, the permanent fraction in the network, at high extension, serves to prevent such a creep, and makes the aligned actuating structures fully reproducible for many heating cycles. Our procedure started with deforming (in simplest cases: uniaxial stretching) the polydomain nematic network by about $100 \%$, and then - while keeping the stretched length fixed - increasing the temperature to $80^{\circ} \mathrm{C}$ (just below $\mathrm{T}_{\mathrm{ni}}$ ) (Figure 3a). On such heating the stretched network develops a much higher local tensile stress, but the continuous creep is prevented by the permanent network fraction. After 24 hours, we cool the sample and remove it from the stretching frame, recording a uniformly aligned (optically transparent) xLCE structure that was formed by the partial bond exchange. The optical and mechanical anisotropy are characterized using polarized optical microscopy and wide-angle x-ray scattering (WAXS). Birefringence associated with the uniaxial aligned xLCE can be observed between crossed polarizers (Figure 3b). The WAXS measurement confirms the nematic alignment, Figure 3c. The order parameter $\mathrm{Q}$ was calculated using the Deutsch method: at room temperature $\mathrm{Q}=0.78$ (the simpler but less justified Herman method gives $\mathrm{Q}=0.74) .{ }^{[52,53]} \mathrm{We}$ also used the model of main-chain nematic polymers to calculate $Q$ as a function of temperature from the reversible thermal actuation data in Figure $3 e^{[54,55]}$ The theory calculated the measured actuation strain as: $\lambda=\left(\ell_{\|} / \ell_{\perp}\right)^{1 / 3} \sim \exp [2 \sqrt{\Omega} / 3]$, where the order parameter is related to the effective chain stiffness as: $Q=1-3 / 2 \sqrt{\Omega}$. One can now re-calculate $Q$ from $\lambda$, which is shown in Figure 3d. As expected, the order parameter sharply decreased with increasing the temperature, reaching zero in the isotropic phase (Figure 


\section{WILEY-VCH}

$3 d)$. The nematic to isotropic transition point $\left(T_{n i}\right)$ is experimentally measured using DSC as $\mathrm{T}_{\mathrm{ni}}=90^{\circ} \mathrm{C}$, which matches the results of thermal actuation very well.

To characterize the shape changing behavior of our aligned LCE (15BDB), we preformed cyclic thermal actuation, when the sample with a low load (hanging weight of $4 \mathrm{~g}$ ) is repeatedly heated into the isotropic phase, and then cooled back down to ambient temperature, maintaining a reasonably low heating rate to stay close to equilibrium. Figure $3 e$ demonstrates the relative change of natural length along the director alignment, $\lambda=L(T) / L_{\text {iso }}$, with the maximum actuation strain of almost $100 \%$, and a small hysteresis inevitably the consequence of the first-order nematic-isotropic transition.

To demonstrate the ability of our xLCE to configure into complex actuating shapes, we programmed a dome-like object by pressing a flat polydomain xLCE film into a die mold using a rounded glass rod with a dimeter of $5 \mathrm{~mm}$. Then the sample was placed in an oven at $80^{\circ} \mathrm{C}$ for $12 \mathrm{hr}$ to allow for the alignment to take place around the glass rod, following our generic alignment protocol. Upon removing the mold, the sample adopted the new dome-like shape with optically transparent walls indicating alignment. The dome-like shape (6mm in height) actuates into a flat disk upon heating above $\mathrm{T}_{\mathrm{ni}}$ (Figure 3f, movie 1, Supporting Information). Such shape transformation is fully reversible, and could be useful in designing tactile displays and other interactive applications.

A very reasonable question may arise about how well does the programmed monodomain alignment lasts, in various conditions. After all, the logic says that the highly stretched permanent network component in the partial vitrimer would pull back on the reconfigured aligned exchangeable part of the network - and given that our boronic-ester exchange occurs reasonably fast at all temperatures (see Figure.1b) one might expect a gradual loss of alignment. We have, therefore, investigated this question. First of all, deep in the nematic phase we found no loss of alignment at all: in the 


\section{WILEY-VCH}

equilibrium programmed shape of the body, the returning traction of the permanent network component is compensated by the extended chain configuration (high $\ell_{\|} / \ell_{\perp}$ ). But when the aligned sample is brought towards the isotropic phase, $\ell_{\|} / \ell_{\perp}$ diminishes and the retraction of over-stretched permanent network component becomes relevant. Figure 4a shows the results of the test when the uniformly aligned monodomain 15BDB xLCE was heated into the isotropic phase and kept there for an increasingly long time; after that period, it was cooled back into the nematic phase and the sample length remeasured. It was difficult to test a sample with zero load, but we were able to apply a low stress of $0.6 \mathrm{kPa}$ (at constant weight of $0.5 \mathrm{~g}$ ), and then carry out several increasing constant-stress tests. Clearly, the 'loss of alignment' occurs, in both cases within a characteristic time of 20-30 min (obtained by exponential fitting, shown by solid lines in the plot). The sample with a very low tension essentially recovering its original polydomain length and conformation (also equal to the isotropic length of both samples). When the tensile stress was more noticeable (albeit still quite low), the long-term recovery saturated with a reasonably well-aligned monodomain elastomer, which we regard as the "isotropic-genesis" monodomain, because the sample spent almost 20 hours at $100^{\circ} \mathrm{C}$ under a uniaxial stress. Figure $4 \mathrm{~b}$ illustrates how this final saturated value of remaining alignment increases with the load applied while in the isotropic phase. The fitting line is a guide to an eye, and we may conclude that when a tensile stress of above $10 \mathrm{kPa}$ is kept on the sample, it does not lose any significant alignment while heated into the isotropic phase. This is different from attempting to heat a polydomain sample under a low load: in that case, the domain boundaries were not yet erased (as in our programming procedure), and a very long time would be required to obtain an isotropicgenesis alignment under comparable loads. Note that in the actuation test of Figure 3e, the time spent in the isotropic phase was only a few minutes, so the question of any possible alignment loss does not arise. 


\section{WILEY-VCH}
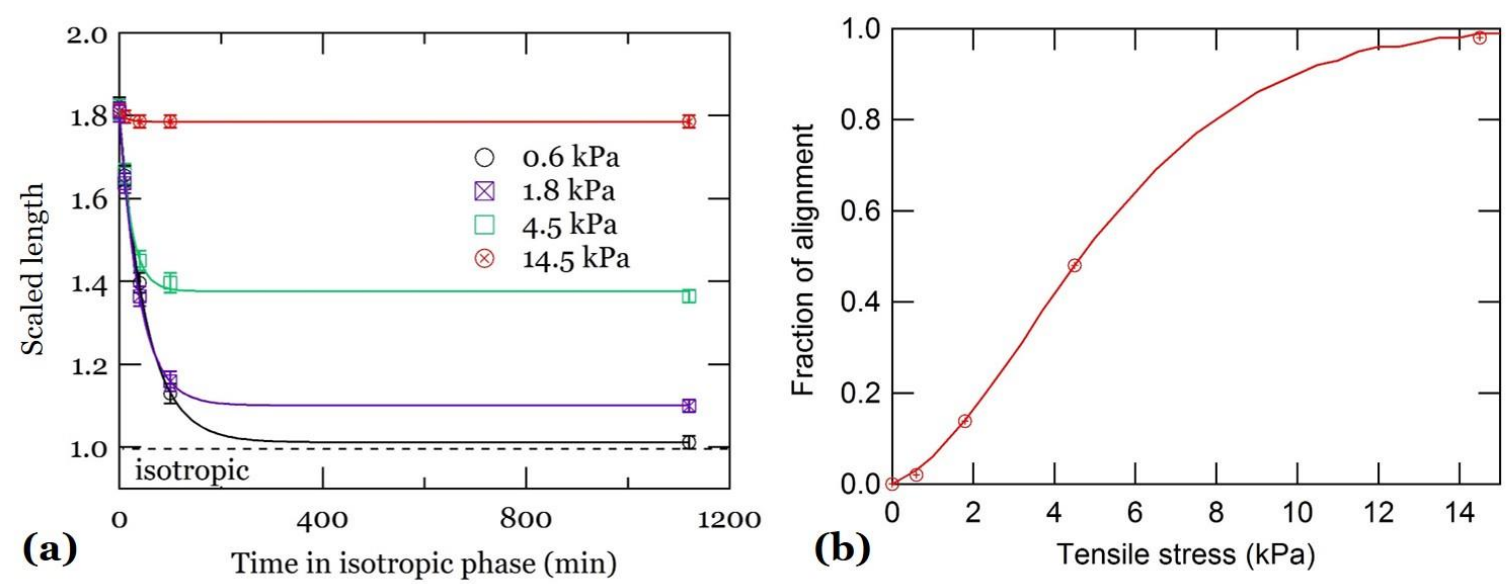

Figure 4. Loss of alignment in the isotropic phase. (a) The natural length of the sample cooled back to ambient temperature, having spent a certain amount of time in the isotropic phase $\left(\right.$ at $100^{\circ} \mathrm{C}$ ), with a constant load applied, labelled in the plot. The lines of exponential relaxation are a guide to an eye, also telling that the characteristic time to lose (all or fraction of) the alignment is $\sim 20 \mathrm{~min}$. (b) The dependence of the final (after 17 hours) saturation value of remaining alignment on the tensile load applied. The solid fit line is a guide to an eye.

As a result of this analysis, we conclude that while a fraction of permanent network in the partial xLCE vitrimers does eliminate the residual creep at high temperatures, the gradual loss of alignment in the load-free isotropic phase is the 'price we pay', because it is this same permanent network pulling back on the exchangeable segments that causes this loss. In many actuator applications this is not an issue: when the 'artificial muscle' remains under some tension in the isotropic phase, no alignment loss occurs. However, in designing applications of such an actuator when the high-temperature state has zero load one should be aware of this effect, and ideally only spend a short time above $\mathrm{T}_{\mathrm{ni}}$, to minimize the loss of alignment. This is almost the opposite of the fully exchangeable xLCE, where any load in the isotropic phase would cause creep, but a load-free state would not be losing its alignment on its own.

It is important to recognize that the entire thermoplastic industry is using remolding and welding to process polymers, with countless applications fields ranging from 


\section{WILEY-VCH}
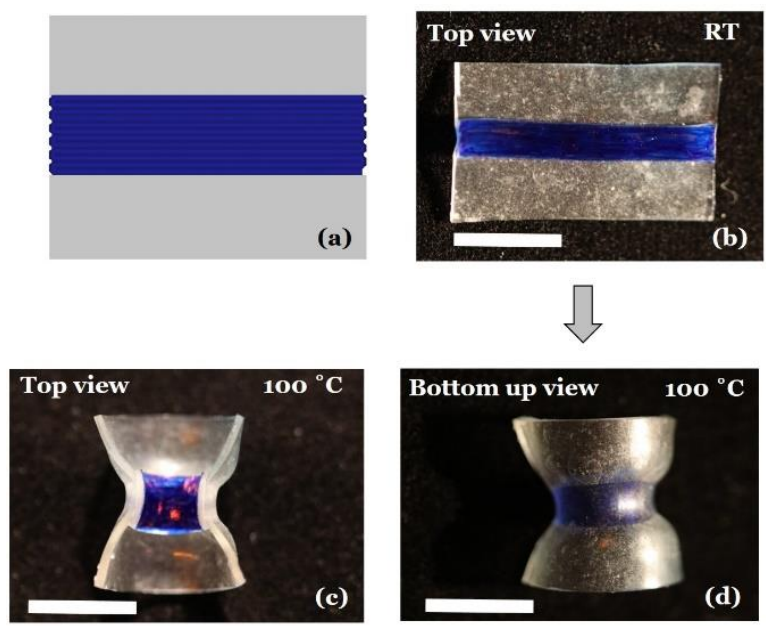
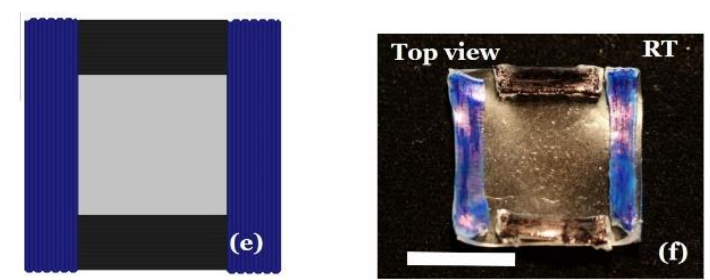

ת
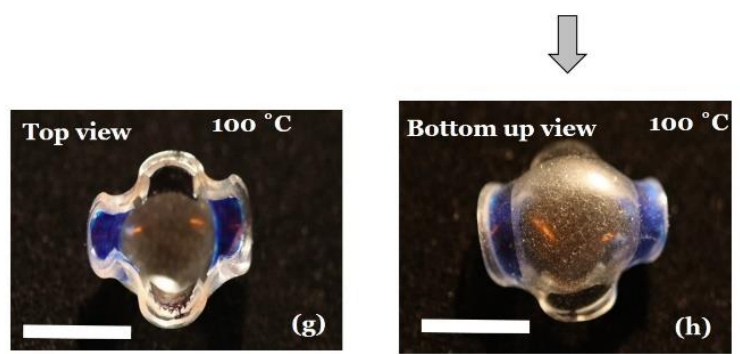

Figure 5. Actuation of multi-layered xLCE composites. a) A scheme of a $22 \times 16 \mathrm{~mm}$ isotropic film welded with a uniaxial aligned (along the $\mathrm{x}$-axis) $22 \mathrm{x} 4 \mathrm{~mm}$ LCE strip. b)c)-d) The shape changes of the bilayer composite film. e) A scheme of a square $16 \times 16 \mathrm{~mm}$ isotropic base layer covalently bonded to four xLCE strips with different director orientations. Two strips $(4 \times 16 \mathrm{~mm})$ are aligned along the y-axis (blue), the other two strips (4x10mm) aligned along $\mathrm{x}$-axis (black). $\mathrm{f})-\mathrm{g})-\mathrm{h}) \mathrm{g}$ and $\mathrm{h}$ ) The shape changes of the bilayer composite (top and bottom up views showing the complex Gaussian curvature at $\left.100^{\circ} \mathrm{C}\right)$. Scale bar: $8 \mathrm{~mm}$

household to aerospace. Here we demonstrate the manufacturing and assembly capability through remolding and welding of our partial vitrimer materials, to produce shape-morphing actuators. We first covalently bonded a bilayer system that is composed of an aligned 15BDB xLCE strip (top, $0.8 \times 4 \times 22 \mathrm{~mm}$ ) and an isotropic $25 \mathrm{BDB}$ film at the base $(0.8 \times 16 \times 22 \mathrm{~mm})$. The LCE strip is programmed for uniaxial alignment along the $\mathrm{x}$-axis (Figure 5a), and the two materials are welded together following the procedure described above (Figure 5b). The composite film is then allowed to cool slowly to room temperature, the LCE part remaining optically transparent, indicating that the alignment is preserved. Upon heating the structure above $\mathrm{T}_{\mathrm{ni}}$ (to $100^{\circ} \mathrm{C}$ ), the structure morphs into a complex geometry (Figure. 5c and d, movie 2, Supporting Information). Upon heating the structure, the LCE material is expected to contract along the $\mathrm{x}$-axis, but also expand along the perpendicular $\mathrm{y}$-axis due to its incompressibility. 


\section{WILEY-VCH}

The presence of the bonded isotropic layer (of similar modulus and thickness) causes the bilayer structure to morph into a half-tube shape with a negative Gaussian curvature

caused by the mismatch of local deformation in the two layers. ${ }^{[56-58]}$ A bilayer with comparable shape and size but without director alignment (polydomain LCE) did not change shape when heated (Figure S4, Supporting Information).

Further manipulation of bonded elastomers with variable alignment in our bilayer system can lead to formation of new shapes. For example, in Figure 5e and g, we show a bilayer with the $16 \times 16 \mathrm{~mm}$ isotropic base bonded with four xLCE stripes with different direction of alignment: two 4x16mm stripes with orientation along the y-axis (colored blue), and two $4 \times 10 \mathrm{~mm}$ stripes with orientation along the $\mathrm{x}$-axis (colored black). Heating this structure generates high localized deformation with complex curvature (movie 3, Supporting Information).

\section{Conclusions}

In this study, we presented a versatile synthetic route to a class of LCE with a fraction of exchangeable boronic ester bonds, which can be molded into complex shapes, with the stress relaxation and the plasticity carefully controlled. Materials with exchangeable boronic ester bonds are synthesized using a Michael-addition thiol-acrylate reaction. Control of plasticity is achieved by varying the amount of boronic ester-containing monomer, where a material 'sweet spot' with an optimum elastic/plastic balance is identified. Such xLCE allows processing post polymerization, while minimizing the unwanted plastic flow. Moreover, conjoining multiple materials (isotropic and LCE) in a single covalently-bonded composite structure results in a smart morphing bilayer system that adopt shapes with complex curvature. 


\section{WILEY-VCH}

\section{Experimental Section}

Materials: Benzene-1, 4-diboronic acid, 1-Thioglycerol, Magnesium sulfate, and Tetrahydrofuran (THF) are used to synthesize the boronic ester containing monomer according the protocol describes in the supporting information. ${ }^{46}$ Bisphenol A ethoxylate diacrylate (BSPDA), Pentaerythritol tetrakis (3- mercaptobutylate), 2,2'-(Ethylenedioxy) diethanethiol, Triethylamine are used to produce the isotropic networks. All chemicals were purchased from Sigma Aldrich and used as received. 1, 4-bis-[4-(6-acryloyloxyhexyloxy)benzoyloxy]- 2methylbenzene (RM82) is used to create the liquid crystal elastomers and was purchased from Wilshire Technologies. Both isotropic and LCE networks are synthesized using a previously described method ${ }^{47}$

Differential scanning callorimetry (DSC): (DSC4000 PerkinElmer) is used to obtain the transition temperatures. Samples with approximately $10 \mathrm{mg}$ were loaded into standard aluminum DSC pans. The samples were heated to $125^{\circ} \mathrm{C}$ at $10^{\circ} \mathrm{C} / \mathrm{min}$, held isothermally for 5 min to undo the thermal history, and cooled to $-50^{\circ} \mathrm{C}$ at $10^{\circ} \mathrm{C} / \mathrm{min}$. Then samples are heated again to $125^{\circ} \mathrm{C}$ to obtain the data. $\mathrm{T}_{\mathrm{g}}$ and $\mathrm{T}_{\mathrm{ni}}$ can be found at the step change in the slope of the heat flow signal and $\mathrm{T}_{\mathrm{ni}}$ can be obtained at local minimum of the endothermic peak. The sample was run three times.

Stress relaxation mesurements: (DMAQ800 TA instruments) is used to characterize the relaxation behavior of boronic ester containing polymers. Samples with dimensions of approximately $15 \mathrm{~mm}$ x $5 \mathrm{~mm} \times 0.9 \mathrm{~mm}$ were tested. All of the samples were tested under constant uniaxial strain $3 \%$ imposed at $\mathrm{t}=0$, the strain was held constant isothermally for 30 min at 40 or $80{ }^{\circ} \mathrm{C}$. Prior imposing the strain, samples were kept at the desired temperature for $5 \mathrm{~min}$. Samples were annealed at $80^{\circ} \mathrm{C}$ for $12 \mathrm{hr}$ before the relaxation test. The normalized equilibrium stress is measured at the plateau of stress vs. time.

Stress-strain mesurements: We used home-made equipment that allowed fine and versatile control of stress-strain-temperature control. All of the stress strain experiments were done with 


\section{WILEY-VCH}

LCE samples $(15 \mathrm{~mm} \times 5 \mathrm{~mm} 0.9 \mathrm{~mm})$ at strain rate of $0.0018 \mathrm{~S}^{-1}$, isothermally at $70{ }^{\circ} \mathrm{C}\left(\mathrm{T}_{\mathrm{ni}}-\right.$ 20). Each test was run three times $(\mathrm{N}=3)$.

Wide angle $X$-ray scattering (WAXS): The phase of the present LCE at RT was characterized using a Philips diffractometer using a Philips Copper target (PW-2233/20) with the wavelength of $0.154 \mathrm{~nm}$. The distance between the sample and the imaging area was $100 \mathrm{~mm}$

\section{Supporting Information}

Supporting Information is available from the Wiley Online Library or from the author.

\section{Acknowledgements}

This work was supported by the European Research Council AdG No. 786659. The authors are grateful for useful communications and advice from Mark Warner and Takuya Ohzono. We would also like to acknowledge Herman Guo and Nicholas Traugutt for helping with experiments.

\section{Conflicts of interest}

The authors declare no conflicts of interest

Received: ((will be filled in by the editorial staff))

Revised: ((will be filled in by the editorial staff)) Published online: ((will be filled in by the editorial staff)) 


\section{WILEY-VCH}

\section{References}

[1] J. Küpfer, H. Finkelmann, Die Makromol. Chemie, Rapid Commun. 1991, 12, 717.

[2] A. R. Tajbakhsh, E. M. Terentjev, Eur. Phys. J. E 2001, 6, 181.

[3] T. J. White, D. J. Broer, Nat. Mater. 2015, 14, 1087.

[4] M. Warner, E. M. Terentjev, Liquid Crystal Elastomers; Oxford university press, 2007; Vol. 120.

[5] S. V Ahir, A. R. Tajbakhsh, E. M. Terentjev, Adv. Funct. Mater. 2006, 16, 556.

[6] M. O. Saed, R. H. Volpe, N. A. Traugutt, R. Visvanathan, N. A. Clark, C. M. Yakacki, Soft Matter 2017, 13, 7537.

[7] J. E. Huber, N. A. Fleck, M. F. Ashby, Proc. R. Soc. London. Ser. A Math. Phys. Eng. Sci. 1997, 453, 2185.

[8] C. P. Ambulo, J. J. Burroughs, J. M. Boothby, H. Kim, M. R. Shankar, T. H. Ware, ACS Appl. Mater. Interfaces 2017, 9, 37332.

[9] Z. Pei, Y. Yang, Q. Chen, E. M. Terentjev, Y. Wei, Y. Ji, Nat. Mater. 2014, 13, 36.

[10] Z. Pei, Y. Yang, Q. Chen, Y. Wei, Y. Ji, Adv. Mater. 2016, 28, 156.

[11] Z. Wen, M. K. McBride, X. Zhang, X. Han, A. M. Martinez, R. Shao, C. Zhu, R. Visvanathan, N. A. Clark, Y. Wang, Macromolecules 2018, 51, 5812. 


\section{WILEY-VCH}

[12] Z. Wang, H. Tian, Q. He, S. Cai, ACS Appl. Mater. Interfaces 2017, 9, 33119.

[13] M. K. McBride, A. M. Martinez, L. Cox, M. Alim, K. Childress, M. Beiswinger, M. Podgorski, B. T. Worrell, J. Killgore, C. N. Bowman, Sci. Adv. 2018, 4, eaat4634.

[14] Y. Li, Y. Zhang, O. Rios, J. K. Keum, M. R. Kessler, RSC Adv. 2017, 7, 37248.

[15] M. K. McBride, B. T. Worrell, T. Brown, L. M. Cox, N. Sowan, C. Wang, M. Podgorski, A. M. Martinez, C. N. Bowman, Annu. Rev. Chem. Biomol. Eng. 2019, 10, 175.

[16] X. Chen, Dam Matheus A., Ono Kanji, Mal Ajit, Shen Hongbin, Nutt Steven R., Sheran Kevin, Wudl Fred, Science, 2002, 295, 1698.

[17] J. Canadell, H. Goossens, B. Klumperman, Macromolecules 2011, 44, 2536.

[18] B. T. Michal, C. A. Jaye, E. J. Spencer, S. J. Rowan, ACS Macro Lett. 2013, 2, 694.

[19] T. F. Scott, A. D. Schneider, W. D. Cook, C. N. Bowman, Science. 2005, 308, 16151617.

[20] B. T. Worrell, S. Mavila, C. Wang, T. M. Kontour, C.-H. Lim, M. K. McBride, C. B. Musgrave, R. Shoemaker, C. N. Bowman, Polym. Chem. 2018, 9, 4523.

[21] M. Capelot, D. Montarnal, F. Tournilhac, L. Leibler, J. Am. Chem. Soc. 2012, 134, 7664.

[22] M. Röttger, T. Domenech, R. van der Weegen, A. Breuillac, R. Nicolaÿ, L. Leibler, 


\section{WILEY-VCH}

Science. 2017, 356, 62-65.

[23] W. Denissen, J. M. Winne, F. E. Du Prez, Chem. Sci. 2016, 7, 30.

[24] D. Montarnal, M. Capelot, F. Tournilhac, L. Leibler, Science. 2011 965-968.

[25] W. Denissen, G. Rivero, R. Nicolaÿ, L. Leibler, J. M. Winne, F. E. Du Prez, $A d v$. Funct. Mater. 2015, 25, 2451.

[26] M. M. Obadia, B. P. Mudraboyina, A. Serghei, D. Montarnal, E. Drockenmuller, J. Am. Chem. Soc. 2015, 137, 6078.

[27] D. J. Fortman, J. P. Brutman, C. J. Cramer, M. A. Hillmyer, W. R. Dichtel, J. Am. Chem. Soc. 2015, 137, 14019.

[28] H. Ying, Y. Zhang, J. Cheng, Nat. Commun. 2014, 5, 3218.

[29] P. Taynton, K. Yu, R. K. Shoemaker, Y. Jin, H. J. Qi, W. Zhang, Adv. Mater. 2014, 26, 3938.

[30] P. Zheng, T. J. McCarthy, J. Am. Chem. Soc. 2012, 134, 2024.

[31] Y.-X. Lu, F. Tournilhac, L. Leibler, Z. Guan, J. Am. Chem. Soc. 2012, 134, 8424.

[32] A. Suzuki, J. Organomet. Chem. 1999, 576, 147.

[33] C. D. Roy, H. C. Brown, J. Organomet. Chem. 2007, 692, 784.

[34] A. P. Cote, A. I. Benin, N. W. Ockwig, M. O’keeffe, A. J. Matzger, O. M. Yaghi, 


\section{WILEY-VCH}

Science, 2005, 310, 1166.

[35] W. Niu, C. O’Sullivan, B. M. Rambo, M. D. Smith, J. J. Lavigne, Chem. Commun. 2005, 4342 .

[36] W. Niu, M. D. Smith, J. J. Lavigne, J. Am. Chem. Soc. 2006, 128, 16466.

[37] J. J. Cash, T. Kubo, A. P. Bapat, B. S. Sumerlin, Macromolecules 2015, 48, 2098.

[38] O. R. Cromwell, J. Chung, Z. Guan, J. Am. Chem. Soc. 2015, 137, 6492.

[39] W. A. Ogden, Z. Guan, J. Am. Chem. Soc. 2018, 140, 6217.

[40] J. Lai, J. Mei, X. Jia, C. Li, X. You, Z. Bao, Adv. Mater. 2016, 28, 8277.

[41] J. J. Cash, T. Kubo, D. J. Dobbins, B. S. Sumerlin, Polym. Chem. 2018, 9, 2011.

[42] D. W. Hanzon, N. A. Traugutt, M. K. McBride, C. N. Bowman, C. M. Yakacki, K. Yu, Soft Matter 2018, 14, 951.

[43] S. W. Ula, N. A. Traugutt, R. H. Volpe, R. R. Patel, K. Yu, C. M. Yakacki, Liq. Cryst. Rev. 2018, 6, 78 .

[44] C. M. Yakacki, M. Saed, D. P. Nair, T. Gong, S. M. Reed, C. N. Bowman, RSC Adv. 2015, 5 .

[45] N. A. Traugutt, R. H. Volpe, M. S. Bollinger, M. O. Saed, A. H. Torbati, K. Yu, N. Dadivanyan, C. M. Yakacki, Soft Matter 2017, 13. 


\section{WILEY-VCH}

[46] Y. Chen, Z. Tang, X. Zhang, Y. Liu, S. Wu, B. Guo, ACS Appl. Mater. Interfaces 2018, 10, 24224.

[47] M. O. Saed, A. H. Torbati, C. A. Starr, R. Visvanathan, N. A. Clark, C. M. Yakacki, J. Polym. Sci. Part B Polym. Phys. 2017, 55, 157.

[48] S. M. Clarke, E. M. Terentjev, Phys. Rev. Lett. 1998, 81, 4436.

[49] F. Meng, R. H. Pritchard, E. M. Terentjev, Macromolecules, 2016, 49, 2843.

[50] S. V Fridrikh, E. M. Terentjev, Phys. Rev. E 1999, 60, 1847.

[51] A. Azoug, V. Vasconcellos, J. Dooling, M. Saed, C. M. Yakacki, T. D. Nguyen, Polymer, 2016, 98, 165.

[52] M. Deutsch, Phys. Rev. A 1991, 44, 8264.

[53] S. V Ahir, A. M. Squires, A. R. Tajbakhsh, E. M. Terentjev, Phys. Rev. B 2006, 73, 85420.

[54] X. J. Wang, M. Warner, J. Phys. A. Math. Gen. 1986, 19, 2215.

[55] R. G. Petschek, E. M. Terentjev, Phys. Rev. A 1992, 45, 930.

[56] C. Mostajeran, M. Warner, T. H. Ware, T. J. White, Proc. R. Soc. A Math. Phys. Eng. Sci. 2016, 472, 20160112.

[57] A. Agrawal, T. Yun, S. L. Pesek, W. G. Chapman, R. Verduzco, Soft Matter 2014, 10, 1411. 


\section{WILEY-VCH}

[58] J. M. Boothby, T. H. Ware, Soft Matter 2017, 13, 4349. 